\title{
Transatlantica
}

Revue d'études américaines. American Studies Journal

$2 \mid 2011$

Sport et société / Animals and the American

Imagination

\section{Une balle blanche dans un roman noir}

Le base-ball au cœur de l'enquête dans Squeeze Play de Paul Benjamin (Paul Auster)

\section{Sophie Vallas}

\section{OpenEdition}

\section{Journals}

Édition électronique

URL : https://journals.openedition.org/transatlantica/5482

DOI : 10.4000/transatlantica.5482

ISSN : 1765-2766

Éditeur

Association française d'Etudes Américaines (AFEA)

Référence électronique

Sophie Vallas, « Une balle blanche dans un roman noir », Transatlantica [En ligne], 2 | 2011, mis en ligne le 30 mars 2012, consulté le 04 février 2023. URL : http://journals.openedition.org/transatlantica/5482 ; DOI : https://doi.org/10.4000/transatlantica.5482

Ce document a été généré automatiquement le 4 février 2023

\section{cc)}

Creative Commons - Attribution - Pas d'Utilisation Commerciale - Pas de Modification 4.0 International - CC BY-NC-ND 4.0

https://creativecommons.org/licenses/by-nc-nd/4.0/ 


\title{
Une balle blanche dans un roman noir
}

\author{
Le base-ball au cœur de l'enquête dans Squeeze Play de Paul Benjamin \\ (Paul Auster)
}

\section{Sophie Vallas}

1 Dans plusieurs de ses entretiens, Paul Auster évoque ses années de disette lorsque, jeune poète et traducteur, il se demandait souvent comment joindre les deux bouts. 1978 fut une année particulièrement sombre, souligne-t-il parfois : son mariage s'enlisait et il ne parvenait plus à écrire. Auster tenta alors deux expériences qu'il décrit comme alimentaires:

I invented a game (a card baseball game-which was actually quite good) and spent close to six months trying to sell it. When that failed, I sat down and wrote a pseudonymous detective novel in record time, about three months. It was eventually published, but it only brought in about two thousand dollars, which was hardly the kind of money I had been hoping for. (McCaffery-Gregory 282)

2 Le roman en question, Squeeze Play, fut publié sous le pseudonyme de Paul Benjamin (Benjamin est le second prénom d'Auster) en 1982 et Auster n'en reconnut la paternité que très tardivement, bien après le succès de ses premiers romans ${ }^{1}$.

Il faut croire qu'aux yeux d'un poète américain affamé, le base-ball devait représenter une manne inépuisable, puisque ce roman noir " écrit à toute vitesse » était, tel le jeu de cartes qu'Auster avait imaginé, fondé sur le base-ball. Squeeze Play s'offre donc comme un polar sportif, mélange de deux genres particulièrement populaires aux États-Unis. Si la fiction d'Auster, The New York Trilogy par exemple, s'affirme comme hautement consciente des règles du roman noir mais fermement décidée à les subvertir de façon toute postmoderne, Squeeze Play, au contraire, joue le jeu et s'inscrit résolument dans la veine hard-boiled : l'intrigue est complexe à souhait et contient tous les éléments du genre (parmi lesquels un nombre élevé de cadavres, un boss mafieux flanqué de gros bras menaçants, ou encore une femme aux grands yeux innocents qui se révèle être une beauté fatale) et la voix narrative, celle du privé au cœur du roman, s'essaye à un humour très chandlérien. Parce que son intrigue se déroule dans le milieu 
$\mathrm{du}$ " plus américain de tous les sports », comme on aime à définir le base-ball aux ÉtatsUnis, le roman se classe également dans la catégorie des baseball novels, et même, si l'on en croit Eric Solomon, dans celle des « Jewish-American baseball novels » (52). Il existe une importante littérature critique, aux États-Unis, sur les baseball novels, ainsi que de nombreuses anthologies qui ne cessent de redessiner le genre et de récapituler combien d'écrivains américains (et non des moindres, puisqu'ils incluent, parmi tant d'autres, Bernard Malamud avec The Natural, Robert Coover avec The Universal Baseball Association, Philip Roth avec The Great American Novel, ou encore, très récemment, Don DeLillo avec Underworld ou Dennis Lehane avec The Given Day), y ont apporté leur contribution. L'erreur à ne pas commettre, bien entendu, serait de penser qu'un baseball novel est un roman à propos du base-ball, qui parle de base-ball. Nombre de spécialistes ont expliqué que dans un tel roman, le base-ball, au cœur de l'intrigue, n'est pourtant utilisé que comme une métaphore permettant à l'auteur d'éclairer autre chose, comme un sujet autorisant et célébrant le jeu avec le lecteur. Tom LeClair, par exemple, a ainsi suggéré que si la littérature est un jeu, alors toute fiction fondée sur le sport peut être vue comme offrant une double occasion de jouer, le terrain (de jeu) idéal de la métafiction. Avec Squeeze Play, Auster qui, rappelons-le, n'avait pas encore écrit à l'époque un seul texte en prose, signait sous pseudonyme un roman qui empruntait à deux genres très codifiés, mais aux possibilités narratives prometteuses. Quel mélange parvenait-il à opérer entre roman noir et baseball novel ? Comment le base-ball, inscrit au cœur du roman, fonctionne-t-il dans l'intrigue policière?

\section{Squeeze Play : base-ball, politique et adultère}

Comment présenter ce roman au titre énigmatique pour le lecteur profane, mais prometteur pour le fin connaisseur de base-ball (on y reviendra) ? L'intrigue est en effet si complexe, et en même temps si intrinsèquement liée à une stratégie de base-ball sur laquelle tout le roman repose, qu'il paraît indispensable d'en rappeler ici les grandes lignes. Max Klein, un privé désargenté comme il se doit, récemment divorcé et traînant sa solitude dans les rues de New York, est embauché par une ex-star du baseball, George Chapman, qui vient de recevoir une lettre de menace anonyme. Cinq ans après qu'un accident de voiture a mis fin à une brillante carrière dans l'équipe des New York Americans en le privant de sa jambe gauche, Chapman, à 33 ans, est sur le point de se reconvertir dans une carrière politique en se présentant aux élections sénatoriales sous l'étiquette démocrate. La lettre anonyme, qui fait allusion à l'accident, rappelle un accord à tenir et menace ouvertement son destinataire. Klein commence par s'intéresser à l'accident de voiture de Chapman et découvre qu'il n'était pas fortuit, mais arrangé par Victor Contini, un mafieux auprès de qui Chapman était endetté. Avant de pouvoir aller plus loin dans son enquête, néanmoins, Klein semble d'emblée perdre la partie: Chapman, qu'il était censé protéger d'une menace inconnue, est empoisonné dans son luxueux appartement, et sa magnifique épouse, Judith (que Klein a rencontrée et qui l'a, bien évidemment, profondément troublé), est arrêtée pour meurtre.

5 La mission de Klein consiste donc désormais à découvrir la vérité sur la mort de Chapman et d'innocenter sa jolie veuve dans la foulée. Petit à petit, et malgré de nombreuses mises en garde de plus en plus musclées, Klein comprend que Chapman avait, cinq ans auparavant, commis un suicide sportif : peu intéressé par le base-ball 
malgré son talent de natural et l'adulation dont il était l'objet, il s'était mis à parier sur ses propres performances et avait ainsi accumulé des dettes qu'il n'entendait pas honorer. L'accident destiné à l'éliminer lui offrait paradoxalement une forme de rédemption: sa renaissance en homme politique providentiel, encore auréolé de sa gloire passée, le replaçait au fond sur un autre terrain de sport dont, encore une fois, on voulait le faire disparaître. Klein découvre que l'amant de Judith Chapman, le Professeur Briles, un universitaire en vue, co-auteur avec Chapman d'un livre sur le sport et la société, est impliqué dans les deux tentatives de meurtre : proche de Contini, il a laissé l'accident de voiture se produire en espérant que la mort de Chapman libèrerait sa maîtresse ; puis, délaissé par cette dernière, Briles a vendu le secret des paris et de l'accident de Chapman à Charles Light, le propriétaire de l'équipe de baseball qui haïssait Chapman parce que ce dernier représentait, à ses yeux, une perversion dans le monde pur du base-ball. Light entendait bien utiliser ces révélations pour briser publiquement Chapman au moment de la campagne électorale. Mais Briles, amoureux transi et jaloux, ne pouvait attendre et a envoyé à Chapman cette lettre anonyme qui lance le roman. Chapman, comprenant que sa réputation ne survivrait pas au scandale sur le point d'éclater, a organisé son suicide de façon à ce que son épouse, dont il connaissait la liaison et qu'il détestait, soit convaincue de meurtre. Lorsque le roman s'ouvre, Klein est donc engagé comme un simple pion dont le rôle sera de témoigner des menaces qui pesaient sur Chapman et de valider ainsi la théorie du complot.

6 Au cours de l'enquête de Klein les cadavres s'accumulent à la suite de celui de Chapman: le cœur de Contini lâche, un témoin de l'accident est liquidé et Briles se suicide. Mais au moment où tout semble résolu, Klein démasque Judith Chapman, devenue sa maîtresse. Manipulatrice de génie, elle a utilisé Briles, son amant éperdu, pour se débarrasser de ce mari froid et infidèle avec lequel elle était censée former le couple le plus glamour du circuit sportif. Épouse perverse, elle était derrière chaque nouvelle action de Briles et les annonçait même à son mari, le regardant avec délectation comprendre que le nœud coulant se resserrait inexorablement. Joueuse dans l'âme, elle l'a donc acculé au suicide, sachant qu'il essayait de l'incriminer dans son dernier geste. Les deux époux se haïssaient à un tel point, comprend Klein, qu'ils se faisaient face à la table du petit-déjeuner au moment où Chapman a avalé le poison acheté par sa femme. Malgré les risques encourus, puisqu'elle savait parfaitement qu'elle serait accusée de ce qui allait apparaitre comme un meurtre, Judith Chapman a misé sa vie à la fois sur le silence de son amant transi et sur les efforts du détective enamouré. Après avoir mis à nu toute son implication dans l'affaire, Klein rejette violemment la séductrice désormais démasquée, et le roman se clôt, laissant le lecteur à bout de souffle après maints rebondissements.

7 L'intrigue de Squeeze Play se déploie donc sur plusieurs plans qui, au fil du roman, s'entrelacent: le monde du base-ball, dans lequel Chapman commet l'impardonnable pour une star adulée puisqu'il gâche son talent en en faisant l'objet de paris insensés ; le monde de la politique, dans lequel seule importe la réputation que l'on peut monnayer en votes sonnants et trébuchants; le monde privé enfin où, loin des projecteurs sous lesquels il est habitué à jouer la partition de l'harmonie parfaite, un couple au faite de la réussite sociale se déchire de la façon la plus sordide qui soit. Passant d'une sphère à l'autre au cours de son enquête, Max Klein va comprendre que toutes sont caractérisées par la notion de jeu. Mais comme le titre du roman l'indique, c'est le base-ball qui l'emporte dans cette équation à trois inconnues. 


\section{Squeeze Play : de la tactique sportive à la résolution de l'enquête}

8 Le titre du roman utilise en effet une expression qui désigne une tactique relativement peu fréquente dans un match de base-ball (il faut que de nombreuses conditions soient réunies pour qu'une équipe décide de la tenter), mais spectaculaire. Cette tactique est généralement utilisée à la fin d'un match difficile, avec un score désespéré, et permet à l'équipe en attaque (à la batte) de marquer un point décisif (ou plusieurs) pour enlever le match en comptant non pas sur son batteur (la star dont tout le stade attend une frappe magique et un homerun), mais sur ses coureurs, plus discrets.

Cette manœuvre, Klein la décrit longuement dans les dernières pages du livre. En compagnie de Richie, son fils de neuf ans, il assiste à un match opposant les New York Americans (l'ancienne équipe de Chapman que soutiennent les Klein père et fils) contre l'équipe de Détroit. À la neuvième et dernière manche, les jeux semblent faits : Détroit mène 2 à 0 et les New York Americans ne paraissent pas pouvoir remonter la pente, ce qui afflige Richie. "What happened was totally unexpected », explique alors Klein. "I had seen the play only once before, fifteen years ago when I was playing for my high school team and we lost an important game when the other team pulled it on us. [...] It was the suicide squeeze, and when it's executed properly there's no way to stop it " (185). Dans un squeeze play, l'équipe doit être parfaitement coordonnée pour déployer sa tactique en deux temps : le batteur de l'équipe en attaque (dans la scène du roman, les New York Americans), qui doit être particulièrement habile, renonce à frapper la balle de toutes ses forces et doit au contraire calculer un amorti (a bunt), la dégageant ainsi mollement sur le côté, près du monticule sur lequel se tient le lanceur de l'équipe adverse, puis courir comme il se doit vers la première base. Le lanceur de Détroit ramasse donc la balle et l'envoie logiquement vers la première base avant que le batteur, devenu coureur, n'y parvienne - ce que le défenseur peut faire aisément, éliminant ainsi le batteur. Mais pendant ce temps, un autre coureur a marqué un point : dans le squeeze play, en effet, un coureur de l'équipe attaquante (Americans, donc) doit se trouver en troisième base et, pendant que son batteur effectue son petit numéro et occupe les adversaires avec son amorti, il doit s'élancer aussi vite que possible et rejoindre le marbre (the plate, dernière des quatre bases à atteindre pour accomplir le tour du terrain). L'idée est donc d'avoir un excellent batteur capable d'amortir n'importe quel lancer et de se sacrifier, et un coureur particulièrement rapide qui démarre au quart de tour et vole jusqu'au marbre.

Dans le roman, la manœuvre comporte un degré de stratégie supplémentaire, d'où le terme de suicide squeeze, et même de double suicide squeeze, que Klein emploie, médusé devant l'audace des New York Americans, car l'équipe utilise ici non pas un, mais deux coureurs, l'un placé en troisième base et l'autre en seconde base au début de la manœuvre. Ceux-ci doivent démarrer ensemble au moment précis où la balle quitte la main du lanceur, donc avant même de savoir si l'amorti prévu est réussi, témoignant ainsi d'une confiance aveugle en leur batteur (car si celui-ci ne parvient pas à frapper la balle, les deux coureurs qui se sont imprudemment élancés vers la base suivante sont éliminés), et passer, pour le premier, de la troisième base au marbre et, pour l'autre, de la seconde base à la troisième et enfin au marbre. La notion de suicide dans cette 
manœuvre réside dans le fait que si le coup rate, éliminant simultanément le batteur et les deux coureurs, la manche est terminée et perdue pour l'équipe en attaque :

The moment Wilton [le lanceur de Détroit] went into his delivery to throw the first pitch, you could see it happening. Webster and Turner [les deux coureurs des Americans, respectivement en troisième et seconde base] took off like a pair of gerbils on the basepaths, and Costello [le batteur des Americans] squared around to bunt. Costello laid down a good bunt to the right of the pitcher's mound. By the time Wilton picked up the ball, Webster was crossing the plate [le marbre] with the tying run [point d'égalisation]. Wilton's only choice was to throw to first to get Costello out, and he made a leisurely toss that beat him by three or four steps. What he didn't realize, however, was that Turner had never stopped, and by the time the first baseman saw Turner flying around third on his way home, it was too late. There was a throw, there was a slide, there was a cloud of dust. But Turner was safe, and the game was over. A double suicide squeeze. Three-two Americans and kiss it goobye. They would be talking about that play for the rest of the season. (185)

11 La manœuvre des New York Americans est donc payante (elle rapporte trois points grâce aux trois bases gagnées), et la victoire assurée alors même que l'équipe était menée 2 à 0 . Le gain du match n'intervient donc pas sur un homerun héroïque, qui met en vedette la frappe exceptionnelle du seul batteur, mais grâce au talent combiné de toute une équipe : en faisant d'abord semblant de manquer leur coup, les Americans trompent en fait la vigilance de l'adversaire sur scène, dans la lumière des projecteurs (sur le monticule et le marbre, où se trouvent respectivement le lanceur et le batteur), et déploient leur tactique dans les coulisses (au niveau des deux dernières bases, presque en marge du centre de la scène, d'où détalent des coureurs dont la rapidité doit être exceptionnelle) pour mieux serrer le nœud coulant autour du cou d'une équipe qui n'a rien vu venir.

Dans le métro qui les éloigne de la folie du stade, Klein et son fils revivent le match chacun à leur façon: "[Richie] immersed himself in the Americans' Yearbook I had bought for him at the game, poring over the statistics and pictures with the unbroken concentration of a medieval scholar in the Princeton library » (186). Aux côtés de son fils qui vient de recevoir une magistrale initiation à la stratégie inhérente au base-ball et qui compulse consciencieusement ses sources, Klein traverse une véritable épiphanie qui lui permet soudain de résoudre l'affaire Chapman :

It was on that subway car that everything finally became clear to me. An odd scrap of memory lodged in my mind, a few loose stones fell out of the wall I had been staring at for the past four days, and finally I was looking at the daylight on the other side. [...] I had ventured out looking for pious truths and all-encompassing answers, and I had discovered that the only things that really mattered were of no apparent consequence-the remarks of a ridiculous cab driver and a piece of unorthodox strategy in a baseball game. Everything I had struggled to find out, all the supposedly important information I had fought for and risked my neck for, turned out to be mere details. The lessons I needed to learn had been given to me free of charge. J. Daniels had proved me that things are sometimes only what they seem to be, and the double suicide squeeeze play had demonstrated that the bunt can sometimes be as powerful as the homerun. It had taken me a while to decipher these messages, to read them correctly as metaphors of the case. I had wanted facts, nothing but cold, hard reality, and now I had understood the most important fact of all-that reality doesn't exist without the imagination to see it. (186)

13 La première leçon à laquelle Klein fait allusion lui a été donnée au début du roman par un chauffeur de taxi, J. Daniels, et consiste en une devinette qu'il pose à tous ses clients : à quoi correspond le «J.» dans son nom? Alors que la grande majorité des 
clients proposent «Jack», le chauffeur hurle de rire et explique que ses parents l'ont tout simplement baptisé « J. ». Il n'y a rien derrière l'initiale, rien derrière la façade. La seconde leçon est dispensée par l'équipe de base-ball qui ne cherche pas la victoire dans la gloire éclatante du homerun, mais au contraire prend le risque d'organiser ce qui semble être un double suicide pour mieux berner l'équipe adverse et toucher au but. L'objectif est atteint, l'adversaire est vaincu, grâce à une tactique indirecte et $a$ priori perdante, qui a demandé en réalité une minutieuse combinaison d'éléments.

Le match de base-ball fournit donc au détective dans l'impasse la clef du mystère : il comprend qu'il ne s'agit pas pour lui de chercher qui a assassiné Chapman, qui aurait $\mathrm{pu}$ accomplir frontalement cet incroyable homerun, mais bien de décomposer le meurtre, l'action, et de dénouer le nœud complexe des jeux de pouvoir entre plusieurs personnages impliqués. Comme le naïf Captain Delano dans «Benito Cereno" de Melville $^{2}$, Klein occupe la position d'un voyeur (position qui lui a été assignée par Chapman) paradoxalement aveugle et confronté à un jeu pervers de domination sadomasochiste : il a été dupé par la majorité des acteurs dans cette comédie sentimentalopolitico-sportive, n'a pas su voir derrière les apparences ni remettre en cause des présupposés (un homme qui lui demande sa protection ne peut pas se suicider, une femme belle et sensible ne peut pas être coupable), n'a pas eu "l'imagination" nécessaire pour percevoir le rôle des uns et des autres, jusqu'au moment où les masques tombent. Grâce à la métaphore offerte par le match de base-ball les yeux de Klein, comme ceux de Delano, s'ouvrent au tout dernier moment. L'affaire Chapman ne consiste pas en un meurtre, mais en une multitude de suicides : double suicide (sportif puis physique) de Chapman, bien sûr, mais aussi suicide de Briles qui a, par deux fois, participé indirectement à des tentatives d'élimination de Chapman avant de quitter la scène, en espérant ainsi sauver sa maitresse qu'il aime encore, et subtil suicide de Judith Chapman, enfin, qui se laisse accuser de meurtre mais mise sur Briles et Klein pour être innocentée in extremis et renaitre en veuve éplorée enfin débarrassée de son mari tant haï... Chacun des protagonistes de ce triangle que l'on n'ose pas dire amoureux doit, à un moment ou un autre, se mettre en péril, organiser sa propre disparition comme une étape nécessaire vers l'objectif qu'il s'est fixé - pour atteindre la base suivante qui les rapprochera du marbre. Ce que Klein doit découvrir, c'est donc comment la balle a circulé entre les différents protagonistes de ce match sordide.

\section{La trahison d'un rêve: « field of dreams »}

Car derrière l'affaire Chapman, c'est avant tout la trahison du monde même du baseball que Klein entrevoit. Avant la résolution finale de l'intrigue permise par la fin du match, le roman ménage de nombreux passages dans lesquels l'enquête est apparemment mise de côté pour laisser la place à une célébration du base-ball. Max Klein, en effet, partage la victoire spectaculaire des New York Americans avec son fils, qu'il emmène pour la première fois voir un match de ligue majeure. «I wanted him to see the whole thing with his own eyes » (182), explique-t-il dans un passage lyrique. "The whole thing ", c'est tout d'abord l'entrée dans le stade qui se mérite après des heures passées dans le métro pour atteindre un quartier sans âme («yet another landscape of bricks, stones and urban blight », 181), puis faire la queue autour de l'enceinte. 
But then you walk up the ramp, and there it is. It's almost impossible to take it all in at once. The sudden sense of space is so powerful that for the first few moments you don't know where you are. Everything has become so vast, so green, so perfectly ordered, it's as if you've stepped into the formal garden of a giant's castle. [...] In the middle of the city you will find yourself enveloped in a pastoral universe [...]. (181)

Entre le conte de fée et la verte prairie mythique, le stade est un espace où la ville s'est métamorphosée comme par enchantement (« everything has become so vast, so green, so perfectly ordered»). "Little by little you begin to adjust», poursuit Klein qui énumère patiemment les moindres détails que les yeux émerveillés de son fils vont enregistrer: «[...] the pristine whiteness of the bases, the symmetry of the pitcher's mound, the impeccably tended dirt of the infield». Dans une interview, Don DeLillo expliqua un jour de façon lumineuse son intérêt pour les jeux: « Games provide a frame in which we can try to be perfect. Within sixty-minute limits or one-hundred-yard limits or the limits of a game board, we can look for perfect moments or perfect structures" (DePietro, 6). Le terrain de base-ball, dont la forme est si simple et si élégante à la fois (on parle d'une forme "en diamant»), est l'un de ces lieux magiques pour les amateurs, plus petit et plus parfait qu'un grand terrain de football rectangulaire par exemple, où les proportions rigoureuses, le dessin méticuleux des lignes, le contraste aigu des couleurs sont garants du rêve.

Field of Dreams est d'ailleurs le joli titre de l'adaptation cinématographique du roman de W. P. Kinsella, Shoeless Joe, dans lequel un terrain de base-ball dessiné au cœur d'un champ de maïs perdu voit les fantômes de joueurs morts depuis des décennies venir frapper quelques balles magiques. Dans Squeeze Play, Klein souligne lui aussi l'attrait presque magnétique du terrain qui transporte le spectateur dans une autre dimension où un seul objet sera le point de mire de milliers d'yeux :

For the next two or three hours the geometry of the field in front of you will hold your attention completely. In the middle of the city you will find yourself enveloped in a pastoral universe watching a white ball fly around in space and dictate the actions of eighteen grown men. Nothing will matter to you more than that ball. It will hold you so completely that when you at last file out and return to the normal world, it will stay with you like the afterglow of a flashbulb that's gone off in your eyes. (181-2)

$\mathrm{Au}$ centre du jeu, donc, se trouve la balle, ce point blanc qui se détache sur le vert de la prairie aussi sûrement que la baleine blanche melvilienne sur l'océan, et dont la couleur éblouissante, elle aussi, reste longtemps imprimée sur la rétine qui l'a contemplée. Cette balle blanche et la courbe infinie qu'elle décrit dans l'espace pastoral ("There she blows!»), Don DeLillo en a fait le point de départ d'un autre mastodonte, Underworld ${ }^{3}$ : «Every baseball carries with it the history of the game, in a mysterious way that you don't find in football or tennis or basketball », explique-t-il dans un entretien (DePietro, 152). C'est bien à cette magie quasi mystique du stade, du terrain et de la balle, que Klein veut initier son fils.

19 Et le match auquel ils assistent ensemble est sans doute d'autant plus riche en enseignements que les New York Americans sortent de leur manche un double suicide squeeze : dans la manœuvre, les yeux de tous restent fixés sur cette balle qui, de façon $a$ priori décevante, est à peine frappée et roule pitoyablement sur le terrain. Ce n'est que dans un second temps que les spectateurs doivent détacher leur regard de cette balle utilisée comme un leurre pour le reporter sur les jambes des coureurs. La balle est 
donc, brièvement, désacralisée et réduite à la fonction d'un leurre, mais elle n'en joue pas moins un rôle décisif dans une action collective.

À la fin de la carrière de George Chapman, c'était le base-ball lui-même qui était devenu un leurre: alors que le public l'adulait, que ses co-équipiers comptaient sur lui, luimême ne croyait plus en rien, ne jouait même plus avec son équipe, avait trahi l'idéal de son sport. En utilisant le « field of dreams » comme un simple casino lui permettant de parier sur lui-même, Chapman a donc trahi un rêve dont Ritchie, dans le roman, est l'innocent porteur.

\section{Chapman : la trahison d'un natural}

21 En pervertissant son talent qu'il transforme en objet de paris, Chapman est en effet le premier à pervertir la notion même de jeu. «Baseball is the great American game, a symbol of all this country stands for, and I'll be damned if I let any player try to destroy it » (160), explique Charles Light, le propriétaire des New York Americans qui n'aura de cesse de punir sa star déchue. Aux yeux de Light (gardien de la flamme du sport élu) comme de tant d'autres, le péché de Chapman est d'autant plus impardonnable qu'il émane d'un joueur exceptionnellement gâté par la vie. Lorsque Klein se remémore la dernière saison de Chapman, il le décrit comme une star absolue, enchaînant les records :

He batted for a .348 average, hit forty-four homeruns, knocked in one hundred thirty-seven runs, and was given the Gold Glove at third base. The New York Americans won it all that year. The division title, the pennant, and the World Series. And when it was all over, Chapman was named the Most Valuable Player in the League. (6)

Il le revoit encore, à vingt-huit ans, grand et séduisant, marié à une femme aussi superbe que sophistiquée, affable avec les journalistes comme avec les gamins quêtant un autographe, et de surcroît diplômé en Histoire, amateur d'art et d'événements culturels. "Chapman [...] was almost too perfect a hero ", résume-t-il, évoquant ce chevalier des temps moderne maître d'une ville qui était à ses pieds : « His picture was printed so often you started seeing his face in your sleep. Even the junkies on the Lower East Side knew his name [...]» (6). Même après la perte de sa jambe, Chapman était parvenu à demeurer dans le cœur de ses supporters, et son autobiographie parue deux ans après sa chute, Standing on My Own, l'avait facilement replacé sous les projecteurs. "If there's one thing America worships more than a celebrity, it's a celebrity who makes a comeback» (8), conclut Klein. Aux yeux du public, Chapman avait vaincu l'adversité, et la carrière politique à laquelle il semblait promis n'était que le prolongement naturel de sa gloire sportive. Comme l'explique l'un des démocrates qui aidaient Chapman à présenter sa candidature aux élections sénatoriales : «George was a natural. He had all the instincts, all the moves. I wouldn't have been surprised to see him run for President one day » (145).

"A natural », un homme béni des dieux pour le sport comme pour la politique. C'est avant tout cette image parfaite, cet espoir que tout le monde plaçait en lui que Chapman déçoit lorsque ses paris insensés sur son propre talent et ses liens avec le milieu mafieux sont découverts. Seul, face à lui-même, incapable d'aimer sa femme ou de lier une véritable amitié sportive, incapable même de trouver du plaisir dans ce sport qui a fait de lui un dieu, Chapman détruit son don en en faisant l'objet d'une 
obsession malsaine : les statistiques de ses performances, le baromètre de son talent, ces chiffres qui enchantent tant ses supporters, deviennent l'arme même de sa propre destruction. Les véritables amoureux du base-ball ne parient pas sur les matchs, note Klein bien avant d'apprendre que Chapman en avait fait un art de vivre. S'ils le faisaient, ironise-t-il, alors Luis Ramirez, le gardien de son parking dont la petite guérite est tapissée d'ouvrages et de magazines spécialisés et qui a une fulgurante intelligence du jeu, serait millionnaire en Californie. «But he was a purist, and the idea of making money from baseball upset him. You didn't try to turn an art form into a business. It would kill the pleasure » (52). C'est ce plaisir pur d'un simple gardien de parking ou d'un gamin de neuf ans qui ne se déplace jamais sans son encyclopédie du base-ball sous le bras que Chapman trahit en misant sur sa propre réussite, sur des performances individuelles plutôt que sur le jeu d'une équipe.

Ce que Klein comprend néanmoins rapidement, c'est que l'appât du gain ne jouait aucun rôle dans l'étrange perversion de Chapman. Ce jeu que Chapman jouait avec ou contre lui-même n'était rien d'autre qu'une forme de suicide :

This was genuine madness, a pure and deliberate attempt to destroy himself. Chapman had tried to take control of his talent by pushing it so far that no matter what happened it was bound to fail him. He had had one of the most extraordinary seasons a baseball player could have, and yet it had all come to nothing. But in this way Chapman had gained ascendancy over the monster. It did not matter that it would ruin himself in the process. Nothing mattered but coming into possession of himself, if only for an instant. It had been like walking through fire. The pain had made him real. (160)

Dans ce jeu d'orgueil à la fin duquel, tel un Ahab moderne obsédé par sa monomanie, absorbé dans son combat narcissique, Chapman devait perdre une jambe, l'enjeu est une étrange et auto-destructrice quête de soi. Chapman ne jouait plus contre une équipe ni même avec la sienne, mais contre lui-même, contre sa propre image de champion imbattable. "He took that ol' game of baseball mighty serious ", se souvient un ancien co-équipier, « sort of like it wasn't a game no more. I don't think he ever got much fun out of it, he was always concentrating so hard » (147).

Le héros d'Auster incarne, au fond, la perversion d'un paradoxe au cœur même du baseball : sport d'équipe, le base-ball n'en aime pas moins construire des héros qui éclipsent leurs partenaires, des Joe DiMaggio, des Lou Gehrig ou des Jackie Robinson, sans parler de ceux qui reçurent un surnom inséparable de leur gloire - Shoeless Joe Jackson ou Babe Ruth. Le jeu est collectif, bien sûr, mais dans les gradins, les gamins qui collectionnent les cartes de leurs héros et leurs pères qui compulsent à longueur d'année les statistiques et autres records dont le base-ball est plus friand que n'importe quel autre sport, attendent tous le joueur qui va illuminer le match par un homerun de légende. À la fin du film tiré de The Natural, le roman de Malamud qui incarne sans doute le mieux le classical baseball novel, Robert Redford, sans doute l'acteur le plus typiquement américain du moment, choisi pour incarner le plus américain de tous les héros sportifs, offre ainsi à son public une frappe inouïe et un homerun inoubliable, et court au ralenti sous une pluie d'étincelles provoquée par l'explosion des projecteurs. Combien de petits garçons (et de plus grands) ont rêvé devant ce Robert Redford mythique, sans d'ailleurs savoir, la plupart du temps, qu'il trahit le héros beaucoup plus sombre de Malamud? Le base-ball, plus que tout autre sport, offre aux États-Unis ces héros, vêtus de cet uniforme si simple et si élégant à la fois, qui incarnent l'espoir de toute une nation; ces héros que tous les pères qui offrent un gant de base-ball à leur fils 
donnent en modèles; ces héros dont on partage le culte seulement avec les initiés, comme Richie le fait avec Luis Ramirez, le gardien du parking qui, au fond de sa guérite, détient un savoir magique : «It was like being initiated into a mystical universe of arcane numbers, obscure personalities, and cabalistic strategies, and Richie became hooked. Luis became his Virgil, his guide through this land of Gods, demi-gods, and mortals » (63). Pour tous ces inconditionnels du base-ball, Chapman est donc un traittre. Entre ses mains, la balle blanche est devenue une simple bille jetée sur le plateau tournant de la roulette d'un casino.

Pour Klein, néanmoins, la trahison de Chapman qu'il lui revient de mettre au jour est encore plus douloureuse : à la grande époque des New York Americans, Klein s'était en effet identifié au champion dans une relation gémellaire que l'enquête, cinq ans plus tard, vient relancer.

\section{Le suicide squeeze ou le suicide du double}

Au moment où Chapman était au faîte de sa gloire, Klein, lui, était au plus bas. Dans un long flashback Klein se revoit démissionner du bureau du procureur, où il avait commencé sa carrière d'avocat, après avoir refusé de couvrir une sombre histoire de corruption policière. À ce désastre professionnel s'ajoutait un divorce difficile, et son naufrage semblait sans fin. Au plus profond du gouffre, pourtant, le base-ball lui apportait littéralement une bouffée d'oxygène :

I found myself retreating into my childhood, trying to put some order in my world by immersing myself in a time when life still seemed full of promise. One of the things I started to get interested in again was baseball. The very unreality of it was soothing me. It didn't matter that I was using it as a way to avoid having to face the mess I was in. (7)

Surgi du monde de l'enfance, monde irréel et réconfortant, le base-ball, ses matchs que Klein suivait assidûment, ses résultats qu'il consultait religieusement dans les journaux, remplissaient sa vie. "Chapman interested me more than any of the other players because we had played against each other in college » (7), explique-t-il alors, avant de confesser le complexe processus d'identification qui le liait à la star du moment :

Following Chapman during his great season, I somehow thought of him as my alter ego, as an imaginary part of myself that had been innoculated against failure. We were the same age, the same size, and had been through the same Ivy League education. The only difference was anatomical: he had the world at his feet and the world had me by the balls. When he stepped up to the plate at the Stadium I sometimes found myself rooting for him so hard that I became embarrassed. It was as though his success could save me, and the idea of transferring so many private hopes to another person frightened me. Of course, I had gone a little crazy that year. And Chapman went on doing so well, day after day, that in some sense I suppose he really did keep me from going off the deep end. I probably also hated the guy's guts. (7-8)

Le thème du double, si fréquent chez Auster, s'impose ainsi dès le début de l'intrigue, au moment où, ayant reçu la visite de Chapman dans son bureau de détective privé, Klein se souvient de la gloire passée du joueur de base-ball qui reflétait alors si bien, comme un négatif photographique, sa propre déchéance. Sosie de Klein, Chapman était aussi son double inversé, un autre lui-même en positif, pourrait-on dire, un jumeau parfait, et la réussite de l'un contrebalançait ainsi parfaitement l'échec douloureux de l'autre. Le mot d'esprit grivois au cœur du passage, si typique du détective hard-boiled, 
crée un effet de distanciation ironique, bien sûr, mais il introduit également une dimension sexuelle qui traverse l'ensemble du passage - transfert, pulsions violentes, peur, idolâtrie et haine mêlées... Il y avait, dans son identification à Chapman, un double désir de possession et d'agressivité qui l'effrayait et le sauvait tout à la fois de sa propre tentation suicidaire, comprend Klein rétrospectivement.

31 Le mystérieux et très suffisant Chapman qui, cinq ans plus tard, loue les services de Klein a-t-il le moindre souvenir d'avoir joué contre lui à l'université, ou bien son choix est-il le résultat du hasard ? Ni Klein ni le lecteur ne le sauront jamais. Ce que chacun comprend, c'est qu'il fallait à cet homme sur le point d'organiser son suicide un témoin naïf au courant des menaces qui pesaient sur lui et dont l'enquête aboutirait à la mise en accusation de l'épouse machiavélique. Le dédain avec lequel Chapman traite Klein lors de leur premier (et seul) entretien montre bien le peu de considération qu'il a pour celui qui n'est destiné qu'à être un pion. C'est à ce pion qu'il confie la lettre anonyme qui joue le rôle du leurre dans l'intrigue qu'il a mise au point. L'orgueil de Chapman l'empêche sans doute d'imaginer que le privé aux locaux miteux qu'il vient d'employer ira au-delà du rôle qui lui est assigné et qu'il devinera le chantage secret dont il fait l'objet: " It had to mean that Chapman was being squeezed from the other end, that something else was going on at the same time» (142), suppute Klein lorsqu'il commence à entrevoir le piège qui se refermait sur son client. Chapman ne se doute probablement pas non plus que Klein, renouant avec sa fascination passée pour le champion, flirtera vite avec la belle Judith avant même la mort de son mari, puis prendra la place de ce dernier dans son lit, tombant ainsi dans un second piège.

L'expression squeeze play trouve alors un sens second, dérivé du sens sportif : elle décrit une situation dans laquelle une pression est exercée pour faire céder quelqu'un et obtenir de lui un avantage, bien souvent dans un contexte politique ou sexuel. Dans le cas de Max Klein, la manœuvre du suicide squeeze initiée par le champion de base-ball se double d'une tactique similaire orchestrée par la femme fatale. Le prénom choisi pour Mrs Chapman ne manque pas d'évoquer la somptueuse Judith biblique, veuve pieuse et respectée qui sauve son peuple d'Holopherne, le général à la tête de l'armée des Assyriens assiégeant la Judée. Parée de ses plus beaux atours, Judith se rend sous la tente du général et le charme ; au quatrième jour, alors qu'Holopherne est bien décidé à obtenir davantage de sa belle visiteuse, Judith, qui persiste à refuser tout nourriture et boisson de son hôte, laisse le général s'enivrer, s'endormir, et le décapite. Ramenant la tête d'Holopherne à son peuple découragé, elle lève une armée qu'elle mène à la victoire. Comme la Judith biblique dont elle partage la beauté et l'élégance, Judith Chapman se garde bien de partager la nourriture empoisonnée qu'ingurgite devant elle son mari haï, dont elle obtient, elle aussi, la tête. Judith des temps modernes, elle couche avec les hommes dont elle escompte une dévotion aveugle et qu'elle mène à leur perte, n'hésitant pas à compromettre sa réputation.

À la fin du roman, Klein, qui a résolu le double mystère du double suicide squeeze, comprend donc le double rôle de pantin qui lui a été réservé. Utilisé tout d'abord par le mari pour préserver le secret du suicide que ce dernier vient de commettre en validant la théorie d'un complot et d'un meurtre exécutés par l'épouse, il a également été utilisé par celle-ci qu'il doit laver des accusations dont elle fait l'objet (faux suicide social dans laquelle elle se place elle-même) et la réhabiliter en victime innocente d'un mari démoniaque. Klein a donc été le jouet, la balle, d'un couple pervers qui a fait de lui un complice et un voyeur nécessaires à leurs plans respectifs : sans sa présence et sa 
participation, en effet, le double squeeze play des époux machiavéliques ne fonctionne pas.

\section{Retour au roman noir}

Dans Squeeze Play le base-ball permet à Klein de résoudre l'intrigue ; la manœuvre sur le terrain reflète le jeu pervers qui s'est déroulé entre les Chapman, jeu dans lequel, indirectement, le monde mafieux et le monde politique ont également pris part. Klein est capable de passer du ballet synchronisé des joueurs sur le terrain aux basses manœuvres des protagonistes de son enquête grâce à la gymnastique mentale que développe tout connaisseur du base-ball. Intarissable sur sa propre passion pour ce sport, Auster explique en effet souvent que le base-ball est « un jeu où l'esthétique tient une grande part : les lignes visuelles du terrain, d'une clarté particulière, contribuent à fabriquer des souvenirs tenaces. On peut se remémorer un match d'une manière très vivante et très présente " (Auster-de Cortanze 21). Un match auquel on assiste, poursuit-il, possède à la fois une dimension stratégique et esthétique qui s'imprime dans la mémoire du spectateur. Inversement, le récit écrit d'un match se traduit immédiatement dans l'esprit du lecteur en images et mouvements sur le terrain :

Durant la saison de base-ball, j'ouvre le journal et je commence invariablement par lire la transcription des parties qui se sont déroulées la veille. C'est comme un rituel. Si vous possédez une expérience visuelle et physique de ce sport, vous pouvez, rien qu'avec des colonnes de chiffres, reconstituer toute une partie: ils provoquent des images et en quelques secondes vous vous retrouvez sur le terrain au milieu des joueurs. (Auster-de Cortanze 22)

Klein, qui partage certains des goûts d'Auster lui-même ${ }^{4}$, est parfaitement à l'aise avec cette gymnastique abstraite et superpose ainsi mentalement les mouvements des New York Americans et les manœuvres des Chapman qui obsèdent son esprit.

Mais si l'intrigue a laissé la part belle au sport, à la fin du roman, pourtant, le roman noir et ses codes l'emportent. La résolution du mystère, si brillante sur le terrain de base-ball comme dans la machination des époux criminels, est intellectuellement et esthétiquement gratifiante, mais moralement insatisfaisante : Judith Chapman échappe à tout châtiment et Klein, errant dans les rues de New York au petit matin, conclut que le scandale qui ne manquera pas de détruire la réputation de Chapman, cette réputation que celui-ci avait tant cherché à protéger, n'a finalement plus d'importance: "Not that it could have mattered to him. Wherever he was now, he could go on dreaming his big dreams. He and Briles and Contini, they could all go on dreaming for the rest of time " (202). Les " grands rêves" de Chapman remplacent le " grand sommeil » de Chandler, dont l'ombre n'a cessé de planer sur Squeeze Play, mais le résultat est le même. Le base-ball, au fond, n'est donc que la métaphore choisie pour éclairer une société dans laquelle tout est devenu jeu violent et perverti - un jeu dont le détective lui-même ne ressort pas indemne. Tout au long de son enquête, Klein ne cesse d'ailleurs d'utiliser des métaphores sportives qui suggèrent sans relâche que tout est affaire de règles que l'on maîtrise ou pas, de codes qu'on peut ou non déchiffrer, de coups qu'il faut savoir encaisser et donner, et bien sûr, de victoires et de défaites. Après un passage à tabac dans un poste de police, par exemple, Klein, qui est finalement relâché, comprend que pour le Captain Gorinski, le spectacle n'a été qu'un simple interlude sportif : 
"I'll give you one thing," [Gorinski] said. "You sure know how to take a punch."

It was all a sport for him, and he wanted to thank me for being a worthy competitor. It didn't matter that the game had been rigged, that they hadn't given me a chance. I refused to shake his hand when he held it out to me. (100)

De même, au moment où Klein va rencontrer Contini pour la première fois, après avoir eu à faire à ses hommes de main, il se décrit ainsi dans le bureau du fils de Contini, Chip, un avocat qui sert ici d'intermédiaire : « I felt like a boxer being told the rules by a referee before the first round of a fight. The only thing Chip didn't know was that his father had already gone a few rounds with me in abstentia "(125). Jeune avocat idéaliste au bureau du procureur, Klein avait déjà compris que son travail se réduisait à un jeu auquel il n'était pas prêt à jouer: « [Being a lawyer] is a game, with its own set of rules and procedures, and the only thing that matters is winning. I finally realized that I was wasting my time » (168). Son travail de détective se résume désormais à un autre sport, plus dangereux, car l'équipe adverse est redoutable, ce qu'il entend bien faire comprendre à Chapman qui vient louer ses services: «Investigative work is a dirty business, but so is crime, and it's just as well to let people know that even if they are helped, they are going to get hurt a little too. It's a game which no one wins and everyone loses. The only difference is that some people lose more than others » (13). Le jeu, le sport, les matchs, les scores, les points gagnés et perdus structurent donc une société qui ne semble plus connaître que les règles multiples d'une compétition dans laquelle tous les coups sont désormais permis. George Chapman, qui était l'une des stars incontestées du sport roi, devient au fil du roman le maître d'un jeu dévoyé qui consiste à mettre en jeu non seulement sa vie mais aussi son suicide, puisque sa propre mort devient une carte maîtresse dans sa stratégie : «[...] even in death George is still calling the shots » (170), remarque un Klein abasourdi, encore englué dans son enquête.

Emblématique du sport aux États-Unis et du poids économique, politique et affectif que le sport peut représenter dans ce pays, le base-ball s'offre dans Squeeze Play comme une métaphore de la société américaine corrompue, violente et auto-destructrice. La chute auto-programmée du champion, George Chapman, révèle les coulisses peu glorieuses du spectacle américain par excellence : le terrain en diamant où se joue le match, verte prairie garante d'un rêve originel, se transforme dans le roman en une scène violemment éclairée où se déroule un vulgaire psychodrame sportif, conjugal et mafieux. Courant de base en base sur les traces des héros de son enfance, Max Klein, en bon privé hard-boiled, s'abîme un instant dans la contemplation du miroir tendu par son client narcissique et suicidaire, et voyage jusqu'au bout de la perversion avec les époux Chapman. Faut-il voir dans le brillant double suicide squeeze, collectivement mis en œuvre par les Americans et qui permet à Klein de comprendre l'implacable stratégie des amants diaboliques et de sortir à temps de la double boucle dont il devait être l'une des victimes, le signe que le base-ball survivra néanmoins à ses idoles déchues? Bien qu'il en sache long sur les malhonnêtetés dans lesquelles trempe le sport pour lequel bat son cœur, en effet, Klein initie pourtant son fils à la beauté d'un terrain vert sur lequel vole une balle immaculée. Et comme la plupart des pères américains, il lui transmet un héritage peut-être plus complexe qu'il n'y paraît, The Baseball Encyclopedia, ce " livre de plus de quatre mille pages [qui] est la véritable histoire des États-Unis» (Auster-de Cortanze 22). 


\section{BIBLIOGRAPHIE}

AUSTER, Paul, The Invention of Solitude, New York, Faber and Faber, 1988 [1982].

---, The New York Trilogy:City of Glass [1985], Ghosts [1986], The Locked Room [1986], New York, Faber and Faber, 1987.

AUSTER, Paul et Gérard DE CORTANZE, « Le monde est dans ma tête, mon corps est dans le monde », interview in Le Magazine Littéraire, dossier Paul Auster, n³38, décembre 1995, 18-25.

BENJAMIN, Paul, Squeeze Play, London, Penguin Books, 1990b[1982].

---, Fausse balle [traduction de Squeeze Play par Lili Sztajn], Paris, Gallimard, Série Noire 2295, 1992. COOVER, Robert, The Universal Baseball Association, New York, Random House, 1968.

DeLILLO, Don, Underworld, New York, Scribner, 1997.

DePIETRO, Thomas, Conversations with Don DeLillo, Jackson, University Press of Mississippi, 2005.

KINSELLA, W. P., Shoeless Joe, New York, Houghton Mifflin, 1982.

LECLAIR, Tom, « Two on One: The Universal Basketball Zone », in Aethlon: The Journal of Sport Literature 21.1-2, 2003-04, 93-104.

LEHANE, Dennis, The Given Day, New York, Harper Collins, 2008.

MALAMUD, Bernard, The Natural, New York, Harcourt Brace and Cie, 1952.

MCCAFFERY, Larry et Sinda GREGORY, “An Interview with Larry McCaffery and Sinda Gregory”, in Paul Auster, The Art of Hunger, Los Angeles, Sun \& Moon Press, 1992, 269-312.

MELVILLE, Herman, « Benito Cereno » [1855], The Piazza Tales [1856], Evanston, Northwestern University Press, 1987.

RAYNAL, Patrick, « Sous le signe du polar », in Le Magazine Littéraire, dossier Paul Auster, n³38, décembre 1995, 38-40.

ROBINSON, Phil Alden, Field of Dreams, Universal Studios, 1989.

ROTH, Philip, The Great American Novel, New York, Holt, Rinehart \& Winston, 1973.

SOLOMON, Eric, « Counter-Ethnicity and the Jewish-Black Baseball Novel: The Cases of Jerome Charyn and Jay Neugeboren ", in Modern Fiction Studies, Special Issue: Modern Sports Fiction, vol. 33, $\mathrm{n}^{\circ} 1$, Spring 1987, 49-64.

VALLAS, Sophie, « Reflets de l'auteur dans les textes et paratextes de Paul Auster : entre autofascination et auto-dérision » in Autoscopies, représentation et identité dans l'art et la littérature, Annales de l'université de Savoie, n²4, septembre 1998, 243-57.

\section{NOTES}

1. En France, c'est Patrick Raynal qui, dans un article intitulé « Sous le signe du polar » et publié dans le dossier consacré à Auster par le Magazine Littéraire en septembre 1995, révéla la paternité du roman. Il en avait publié la traduction française, Fausse balle, dans la Série Noire en 1992.

2. L'œuvre de Melville est un intertexte fréquent chez Auster, et l'on verra que Squeeze Play n'hésite pas à jouer avec quelques uns des grands symboles melviliens. 
3. Dans Underworld, c'est le destin mystérieux de la balle officiellement perdue dans le public après un fabuleux homerun final dans le match opposant les Giants aux Dodgers, le 3 octobre 1951, qui lance et construit le récit.

4. Le roman a été écrit en 1978, à l'époque où Auster se sépare de sa femme et de son fils et doit trouver une nouvelle orientation dans son écriture, expérience qu'il raconte dans son essai autobiographique, The Invention of Solitude. Max Klein traverse une expérience semblable dans le roman. Pour un développement sur la façon dont, avant de reconnaître la paternité de Squeeze Play, Auster, a joué avec ce roman mystérieux, l'incluant dans ses premières fictions (notamment dans City of Glass, dans lequel le roman apparaît sous le titre de Suicide Squeeze) et créant un jeu vertigineux de masques autobiographiques, voir Sophie Vallas, "Reflets de l'auteur dans les textes et paratextes de Paul Auster : entre auto-fascination et auto-dérision ».

\section{RÉSUMÉS}

En 1982, Paul Auster fit paraître, sous le pseudonyme de Paul Benjamin, un roman noir intitulé Squeeze Play. Ce roman présente la particularité d'appartenir également au genre du baseball novel, l'intrigue se déroulant en partie dans le monde du base-ball et sa résolution intervenant grâce à une manœuvre rare et spectaculaire (un double suicide squeeze) qui éclaire l'enquête sous un jour nouveau. Cet article se propose d'analyser la place du base-ball dans Squeeze Play: élément essentiel de la structuration du roman, le base-ball permet également à Auster d'étudier le mythe du héros américain déchu dans une société dont toutes les dimensions (sportive, politique, privée) sont gangrenées par la corruption et la perversion. La relation particulière qu'Auster tisse entre roman noir et baseball novel est également étudiée.

In 1982 Paul Auster published a roman noir entitled Squeeze Play under the pseudonym of Paul Benjamin. The novel is also a "baseball novel": its plot essentially deals with a baseball New York team and its former, fallen star, and the final resolution of the mystery intervenes thanks to a revelation brought by a baseball game in which a rather rare and spectacular maneuver (the double suicide squeeze) is used by one of the teams to turn the tables and win the game. This paper analyzes the place of baseball in Squeeze Play: it first appears as an essential element in the very structure of the plot, but it is also, for Auster, a way of questioning the myth of the hero in an American society whose main aspects (sports, politics, private life) are eaten up by corruption and perversion. The links Auster establishes between roman noir and baseball novel are also dealt with.

\section{INDEX}

Keywords : Paul Auster, Paul Benjamin, baseball, baseball novels, roman noir, Squeeze Play

Mots-clés : Paul Auster, Paul Benjamin, base-ball, baseball novel, roman noir, Squeeze Play 
AUTEUR

SOPHIE VALLAS

Université de Provence Aix-Marseille 1 